\title{
Lower respiratory tract infections: Treatment challenges and new therapeutic options
}

$\mathrm{L}$ ower respiratory tract infections (LRTIS) include a number of different entities, ranging from acute bronchitis in a young, previously well person to ventilator-associated pneumonia in an older patient with comorbid illness. At one end of the spectrum, it is clear that antibiotics are unnecessary, while at the other end of the spectrum aggressive combination antimicrobial therapy is required and crude mortality rates of up to $70 \%$ have been reported. Two entities fall between these extremes: acute bacterial exacerbations of chronic bronchitis (ABECB), and community-acquired pneumonia (CAP). Together they account for millions of health care dollars spent, much antibiotic use, and significant morbidity and mortality.

During the past decade we have seen a disquieting increase in the incidence of resistance among respiratory pathogens. The pneumococcus that demonstrated a remarkably stable in vitro susceptibility pattern to beta-lactams in general, and to penicillin in particular, for over 40 years suddenly showed an increase in resistance not only to these drugs but to macrolides and, in some cases other agents as well.

Resistance mediated by beta-lactamase production by Haemophilus influenzae and Moraxella catarrhalis has also increased, and Gram-negative bacilli such as the Enterobacteriaceae have shown an alarming rise in resistance to betalactams, secondary to a variety of plasmid and chromosomally mediated mechanisms.

A recent symposioum titled "LRTIs: Treatment challenges and new therapeutic options" was held on September 28, 1997. An international group of physician scientists presented data on the use of newer fluoroquinolones in general, and grepafloxacin in particular, for the treatment of various respiratory tract infections, with a focus on ABECB and CAP. In vitro susceptibility data, pharmacokinetic and pharmacodynamic modelling data, and clinical trial results were presented and discussed. The role of the newer fluoroquinolones in the management of such infections was highlighted.

This publication brings together the pertinent points of the presentations and allows the reader to review the data and the relevant discussions.

Lionel Mandell MD FRCPC

Division of Infectious Disease, and Department of Medicine Henderson Site, Hamilton Health Sciences Corporation and McMaster University, Hamilton, Ontario 


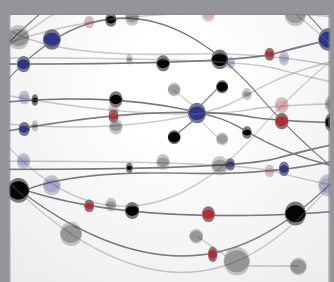

The Scientific World Journal
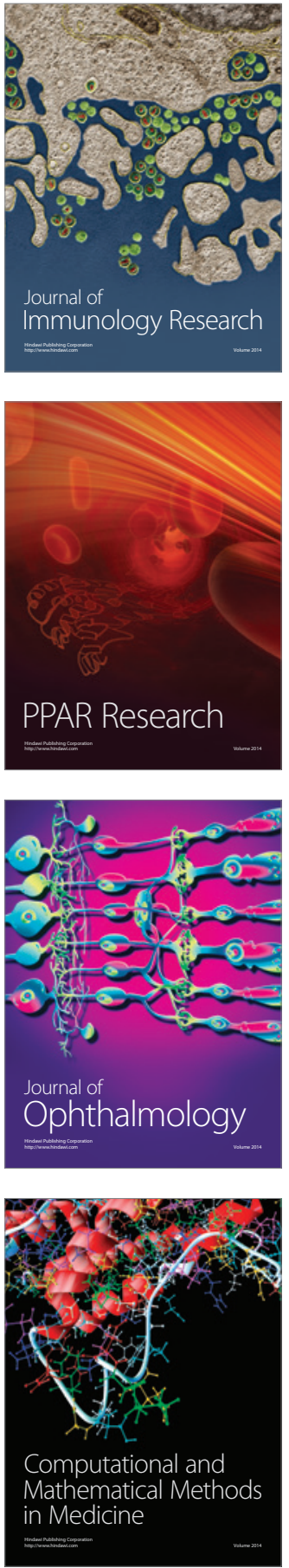

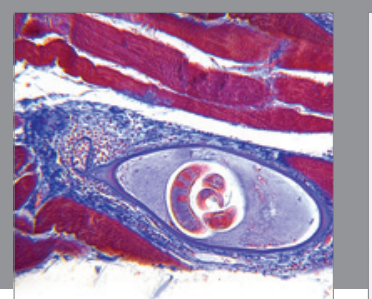

Gastroenterology Research and Practice

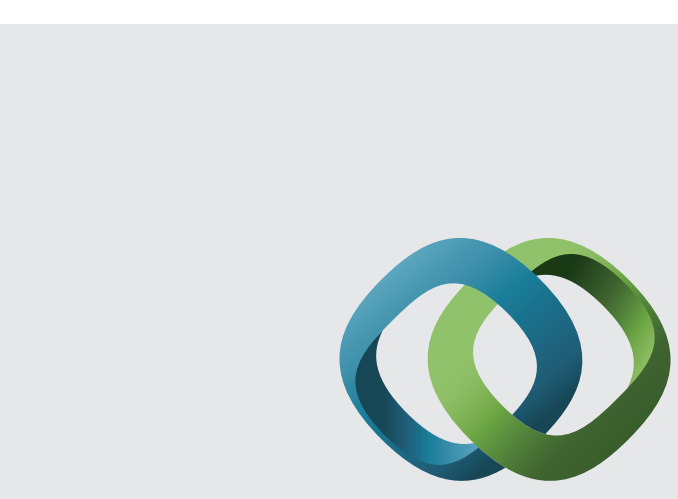

\section{Hindawi}

Submit your manuscripts at

http://www.hindawi.com
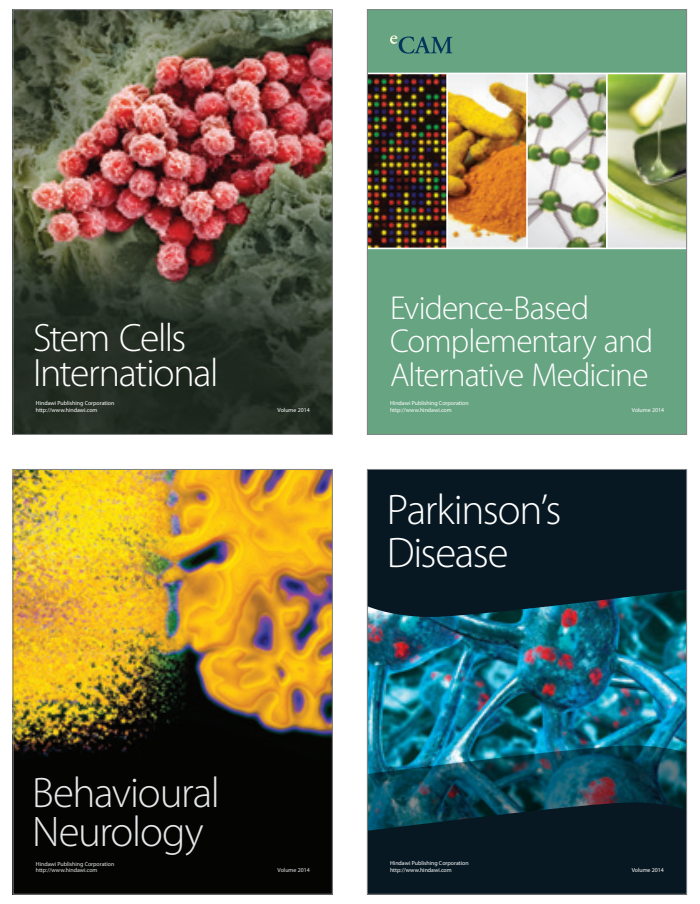
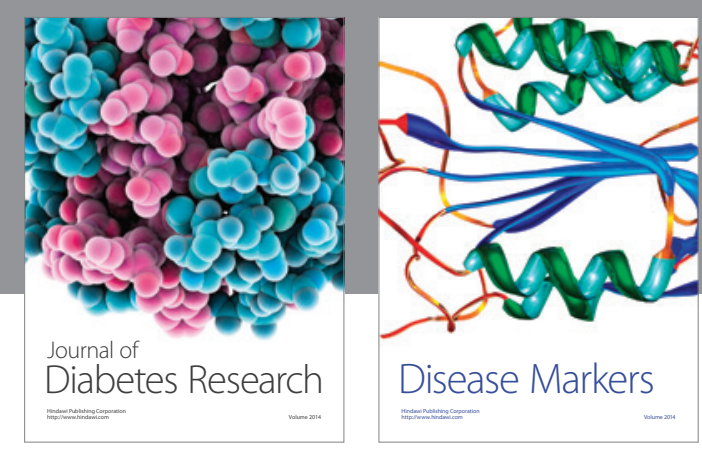

Disease Markers
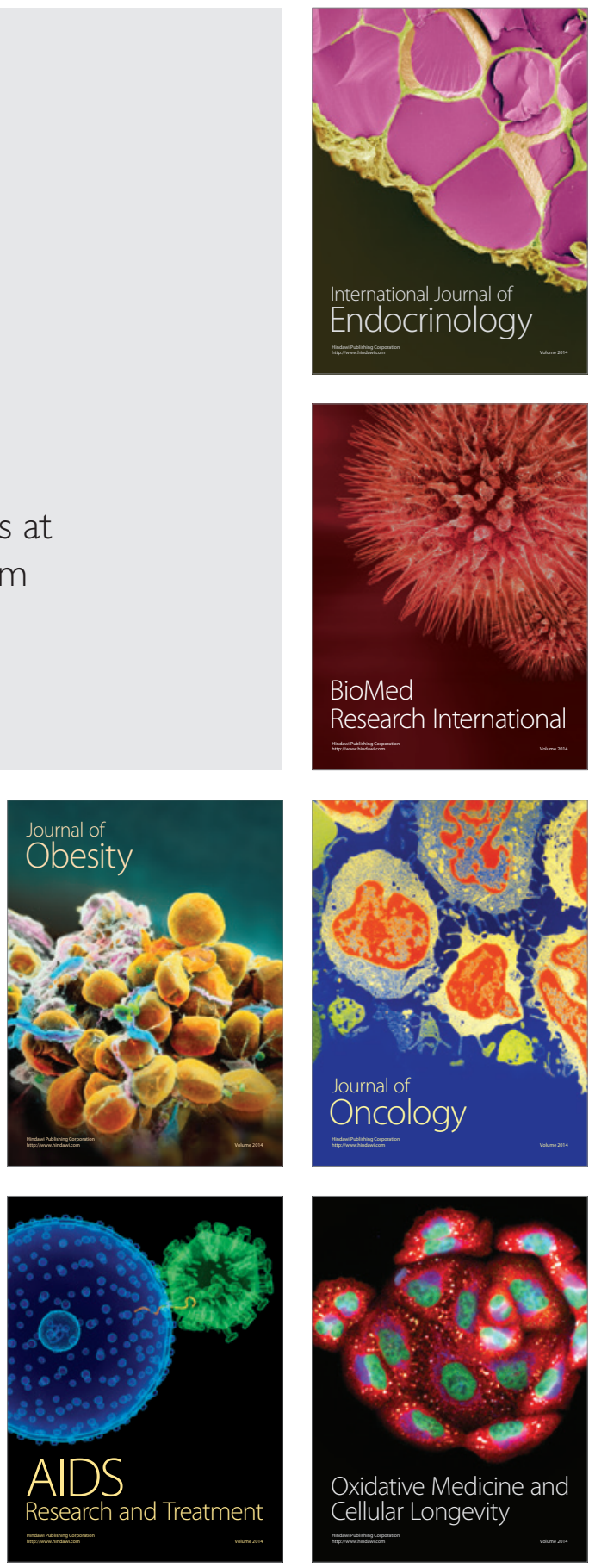